\title{
Risk evaluation for trap geometry in a high structural uncertainty and incomplete velocity data context
}

\author{
Carlos A. Becerra1 ${ }^{1}$, Carlos G. Garzón², María T. Duarte ${ }^{3}$, Alejandro Silva ${ }^{3}$, Iván C. Higuera ${ }^{3}$, William Agudelo ${ }^{3}$. ${ }^{1}$ UIS, \\ ${ }^{2}$ CPVEN, ${ }^{3}$ ECOPETROL.
}

Copyright 2019, SBGf - Sociedade Brasileira de Geofísica

This paper was prepared for presentation during the $16^{\text {th }}$ International Congress of the Brazilian Geophysical Society held in Rio de Janeiro, Brazil, 19-22 August 2019.

Contents of this paper were reviewed by the Technical Committee of the $16^{\text {th }}$ International Congress of the Brazilian Geophysical Society and do not necessarily represent any position of the SBGf, its officers or members. Electronic reproduction or represent any position of the SBGf, its officers or members. Electronic reproduction or
storage of any part of this paper for commercial purposes without the written consent

storage of any part of this paper for commercial
of the Brazilian Geophysical Society is prohibited.

\begin{abstract}
Assessment of prospects in oil and gas industry involves the analysis of multiple information, which allows to have only a partial view about real conditions in the subsurface. Decisions should be making on a short time, and strategies to deal with uncertainty take a key value. This study focuses on trap presence and possible geometry, not on the other elements of the hydrocarbon system. The analysis is performed under the assumption that there have been adequate conditions for oil charge and migration to take place. Our goal is attempting to project the geometry of the structure from avaliable data and assess different seismic velocity scenarios. It can be seen as an alternative solution to deal with uncertainty associated to incomplete data, which allows to generate useful results for improve desicion making process and thereby reducing exploratory risk. A study case with possible traps analyzed from an exploratory block in Colombia is illustrated.
\end{abstract}

\section{Introduction}

It is well know that oil and gas exploration activities are limited in time and data. Time limitations are a consequence of having a highly dinamic industry, while data limitations are due to budget optimizations. Explorers should always deal with uncertainty associated to incomplete data. In the present work we show an study case, where a serie of scenarios were analized in order to deal with uncertainty in the trap's geometry for an exploratory block in the onshore Colombia. The block is located in the Middle Magdalena Valley, between Central and Eastern Cordillera in the Northern Andes. The region has been focus of exploration since forties, with multiples wells drilled since then. There are three small giant oil fields close in the basin. Ecopetrol and CPVEN have explored the block since 2010 .

The exploratory block is partiallty covered by a 3D seismic volume adquiried in 2014 , and by more than 50 2D seismic lines (646 lineal kms) from multiple programs, all of them processed to PSTM. Due to the geological conditions of the study area, where high dips and faultpropagation folding are involved (Figure 1), seismic images are restricted in quality and precision. The geometry interpreted on those seismic images is the main input to identify possible traps; since changes in the geometry of the structure imply changes in the volumen estimation, special attention should be taken over uncertainty related to structure's geometry. Most of the avalaible seimic information is in the time domain, which in general is better in continuity, than information in the depth domain. For that reason, the geometry interpreted in the time domain is used as input for the analysis process. On another hand, velocity information is obtained from wells and seismic. There are three wells located inside the exploration block; however, only one has velocity information. Then, it was to be necessary to use information for additional wells located close to the study area, which provided information for some key intervals. Velocity obtained from seismic processing, is also used as a guide, to make a better extrapolation process, and because it allows to identify the directions in which changes in velocity are given.

As is ilustrated in figure 1, the geometry of the studied area has been established by three main thrust faults, which are (from west to east): faults 3,2 and 1 . In the hanging wall of fault 2 , a cretaceous sequence is present; while a Cenozoic sequence is its footwall, with rocks Oligocene to the present aged. In the northern zone of the studied area, fault 2 splits into two faults called: fault $2 \mathrm{~A}$ and $2 \mathrm{~B}$.

Some possible traps have been identified in the exploratory block. Trap 1 is located in the frontal anticlinal fold related to fault 3 . Trap 2 which is located deeper than trap 1, has closure in hanging wall of normal and inverted faults in the footwall of fault 3.

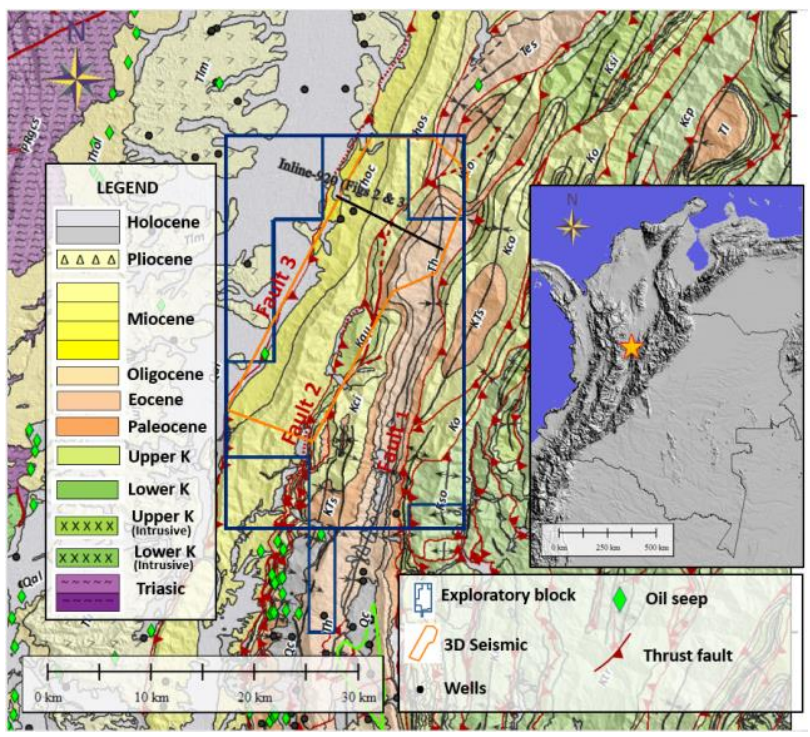


(a)

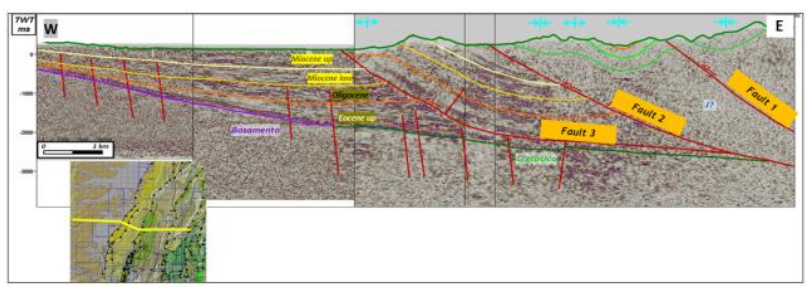

(b)

Figure 1 - (a) Geologic Map displaying main features (Compiled from Ecopetrol-ICP maps), and a (b) generalized cross section in the time domain showing main structural setting in the study area.

\section{Method}

In order to evaluate the geometry of the identified traps in the depth domain, the geometry interpreted in time and the available velocity data have been used to generate velocity scenarios. The definition of those velocity models was based on information of wells as primary source and the seismic velocity as secondary information. The approach used is convenient due to the current available information is not enough to generate a unique velocity model. On another hand, and it is basis on the complexity of the study area, it is expected that the geometry of the structure in the time domain, observed in PSTM images be distorted by velocity effects. Then, we use image ray time to depth mapping method for correcting distortive effects and find the right geometry of the structure.

The velocity scenarios were defined in the time domain, they cover a combination of likely velocities for key stratigraphic units defined using the information available from wells and seismic. It allowed to establish four scenarios represented in the velocity models illustrated in the figure 2.

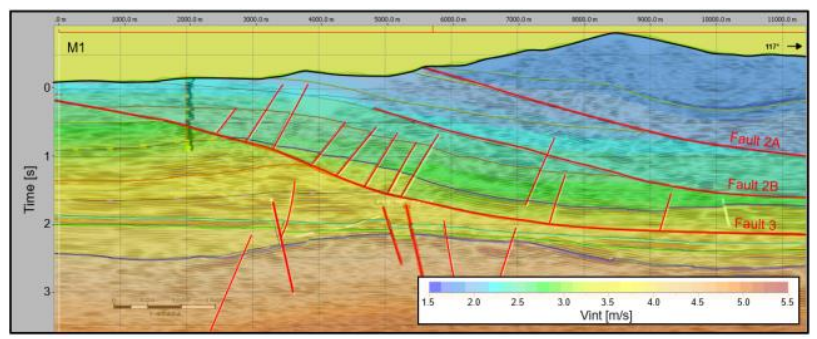

(a)

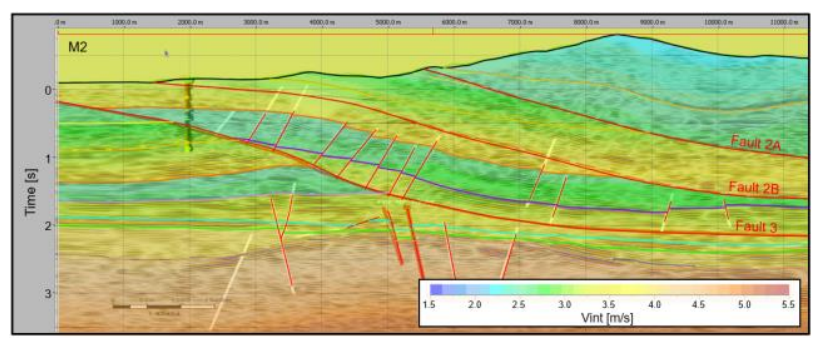

(b)

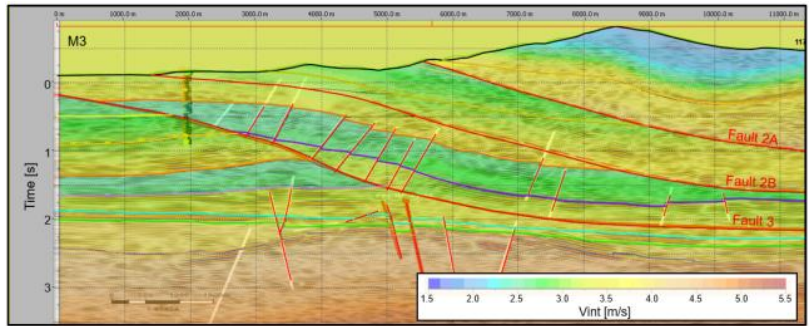

(c)

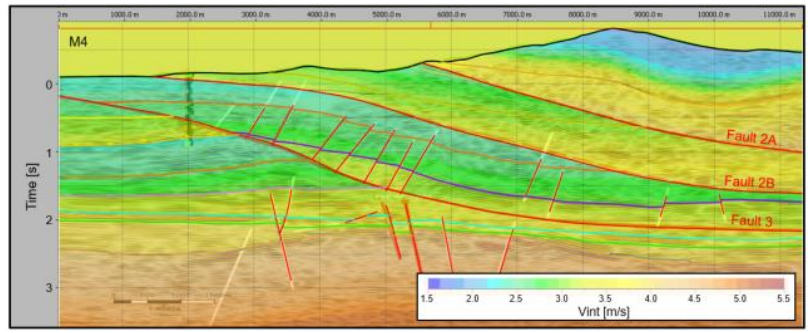

(d)

Figure 2 - Velocity-case scenarios defined by four velocity models ilustrated on inline 902 .

There are many differences between each scenario, in general, Model 1 is the scenario of low velocity, Model 4 the one with the high velocity and Models 2 and 3 represent intermediate cases. Model 1 (M1) has the lowest velocity in the hanging wall of faults $2 \mathrm{~A}$ and $2 \mathrm{~B}$, in comparison with the other models. Only in M1, velocities in the footwall of fault 3 is not correlated with velocities in their corresponding hanging wall. In models $\mathrm{M} 2, \mathrm{M} 3$ and M4, velocities in the hanging wall and in the footwall of fault 3 are correlated; however, M4 represents the scenario with the highest velocities in the footwall of fault 3 in comparison with the other models. Scenarios generation is a way to test hypothesis about the velocity in specific intervals, where information is not completed or has low quality.

Once the scenarios have been defined, the velocity model representing each one should be used to perform image ray tracing and to obtain the geometry in the depth domain. Figure 3 illustrates the mesh used during the image ray tracing process for one of the models in figure 2.

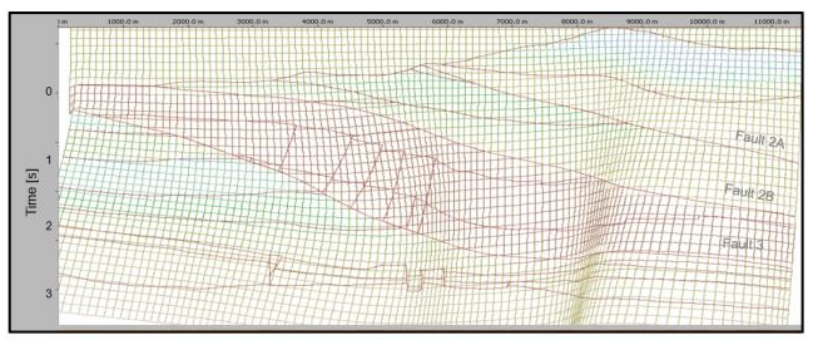

Figure 3 - Image ray tracing illustrated for one of the models on section in figure 2.

This method is employed because it allows to get results without distortions related to strong velocity variations in that domain. Image rays relate the position of reflective events in both, time and depth domain (details in 
Cameron 2007; Iversen and Tygel 2008). They are characterized by being the rays representing the minimal traveltime from a particular point in the subsurface to a free surface, and because they emerge vertically in reference at that free surface. Image ray's curvature is a function of the velocity field, assuming a constant or a vertically inhomogeneous velocity field, curvature should be zero; otherwise, when the properties of the medium change, as usual do, rays present curvature, which is an indicator of changes in the direction of the wavefront when propagation of waves occurs in subsurface. If images rays are traced over a velocity model, the position of each reflective event can be found by computing the distance traveled by the wavefront during half of the time identified on the seismic section in that domain.

\section{Results}

The application of the described method to each model representing a different scenario, allows to obtain four different possible geometries of the analyzed structure in the depth domain. Figure 4 shows the resulting geometry in the depth domain for the models indicated in figure 2.

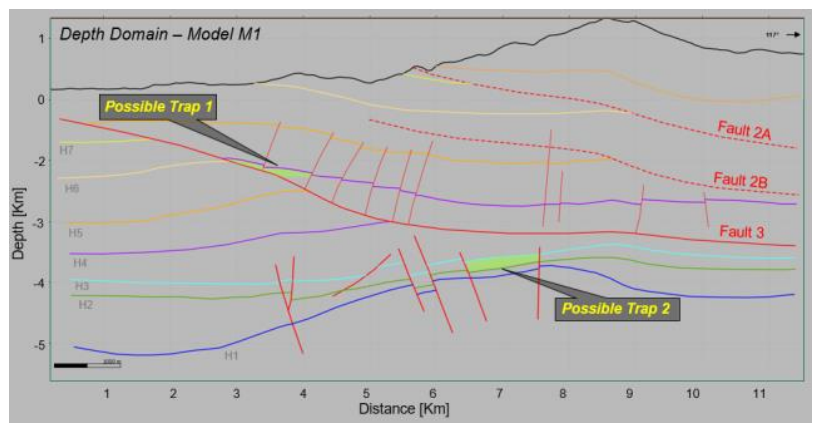

(a)

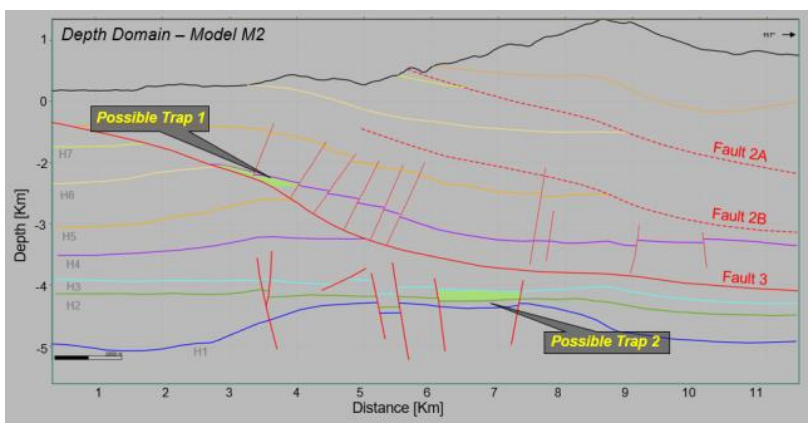

(b)

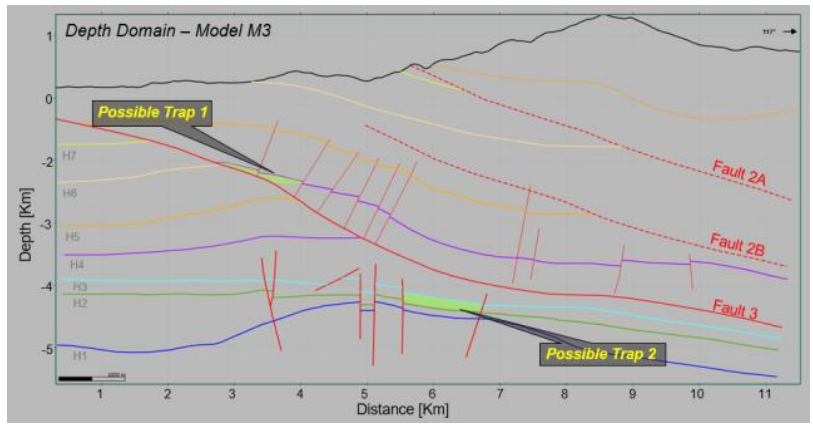

(c)

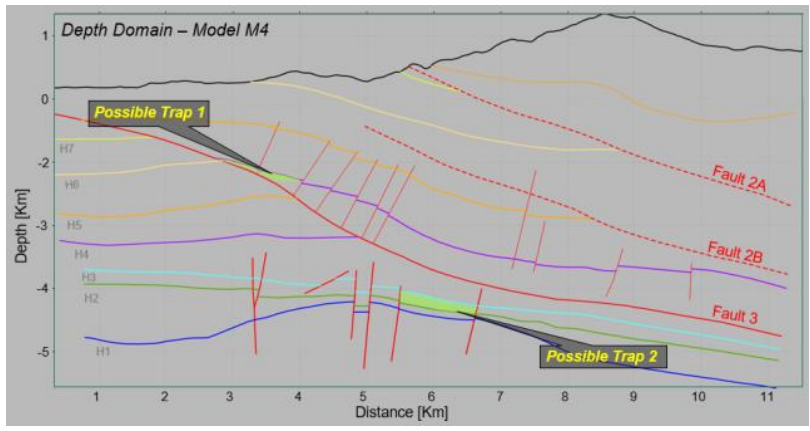

(d)

Figure 4 - Depth domain resulting geometry for each model: (a) Model M1, (b) Model M2, (c) Model M3 and (d) Model M4, representing the set of proposed scenarios.

As is illustrated in the figure 4 , the lower velocity case scenario results in a shallower trap positioning in depth domain. The vertical positioning differences are not significant for trap 1, but for trap 2. That is an indicator of having a higher positioning uncertainty for trap 2. Even more important than trap positioning is the trap geometry, the main aim of the proposed test is to figure it out, if the trap is maintained in all cases. Results indicate that the trap located in the hanging wall of fault 3 , do not loss their structural setting for any of the scenarios analyzed, indicating a low exploratory risk related to the trap existence. The trap geometry remains almost invariant in the case of trap 1.

The possible trap 2 is located deeper than trap 2, and there is more uncertainty related to their positioning and geometry in comparison with the formers. Model M1 indicates a north-west dipping structure, which according with the general structural setting of the basin looks like unlikely. Model M2 suggests a non-dipping structure and on another hand, Models M3 and M4 indicates a southeast dipping structure, a result opposite in orientation to the Model M1. All these possibilities for structure orientation resulting for the analysis to each scenario, is an indicator that there is too much uncertainty involved in the positioning and the geometry of the trap 2. Thus, additional information is required in order to establish their real orientation.

\section{Conclusions}

Based on obtained results for the analyzed scenarios, it was possible to establish the following statements about the possible traps of interest: (1) the likely of trap existence for Trap 1 is high, the structural setting of the trap remained almost invariant in all cases-scenarios analyzed. (2) In the case of the possible Trap 2, a high risk related to the trap existence was identified, it is a fact that the available information is not enough to establish its geometry or its orientation, for that reason this trap was found highly unfeasible.

Results of the study were a useful tool for making decisions in the pre-drilling phase, when any effort in risk reducing means saving money. In addition, the performed 
analysis resulted useful for the decisions making process about future traps in the footwall.

\section{Acknowledgments}

Authors would like to thank Ecopetrol-VEX and CPVEN for their permission to publish results. Also, thanks to Andres F. Vargas for providing interpreted data which allowed to complete the present study case.

\section{References}

Cameron, M., 2007, Seismic velocity estimation from time migration: Inverse Problems, 23, 1329-1369.

Iversen, E., and Tygel, M., (2008), Image ray tracing for joint 3D seismic velocity estimation and time-to-depth conversion, Geophysics, vol. 73, No 3, S99-S114. 